\title{
Packing similarities and synthon variabilities in aminopyridinium sulfoisophthalates
}

\author{
V. Videnova-Adrabinska ${ }^{a^{*}}$, A. Nowak ${ }^{a}$, J. Janczak ${ }^{b}$

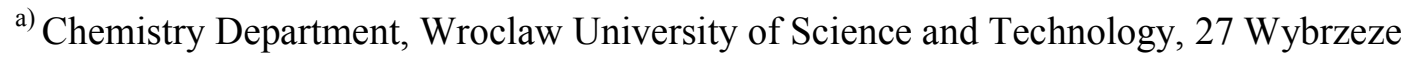 \\ Wyspianskiego St., 50-370 Wroclaw, Poland \\ b) Institute of Low Temperature and Structure Research, Polish Academy of Sciences, \\ P.O.Box 1410, 50-950 Wrocław, Poland
}


Table S16. Dehydration and melting temperatures in crystals 1-5

\begin{tabular}{|c|c|c|c|c|c|}
\hline & \multicolumn{2}{|c|}{ Dehydration Region } & \multicolumn{2}{|c|}{ Melting Region } & \multirow[t]{2}{*}{ Decomposition region } \\
\hline $\mathrm{H}_{3} \mathrm{SIP}$ & & & $>300$ & & \\
\hline 2-apy & & & $59-60^{\circ} \mathrm{C}$ & $\left(T_{\text {boil }} 210^{\circ} \mathrm{C}\right)$ & \\
\hline 3-apy & & & $65^{\circ} \mathrm{C}$ & $\left(\mathrm{T}_{\text {boil }} 248^{\circ} \mathrm{C}\right)$ & \\
\hline 4-apy & & & $155-158^{\circ} \mathrm{C}$ & $\left(T_{\text {boil }} 273^{\circ} \mathrm{C}\right)$ & \\
\hline 2,6-dapy & & & $117-122^{\circ} \mathrm{C}$ & $\left(\mathrm{T}_{\text {boil }} 249^{\circ} \mathrm{C}\right)$ & \\
\hline \multirow[t]{3}{*}{ Crystal 1} & Peak max: & $194.5\left({ }^{\circ} \mathrm{C}\right)$ & Peak max: & $264.7\left({ }^{\circ} \mathrm{C}\right)$ & $\mathrm{T}:>350\left({ }^{\circ} \mathrm{C}\right)$ \\
\hline & Onset: & $178.4\left({ }^{\circ} \mathrm{C}\right)$ & Onset: & $254.4\left({ }^{\circ} \mathrm{C}\right)$ & $\Delta \mathrm{m}(\%): \quad-24.4$ \\
\hline & Offset: & $203.1\left({ }^{\circ} \mathrm{C}\right)$ & Offset: & $290.6\left({ }^{\circ} \mathrm{C}\right)$ & $\Delta m(\%):-8.5$ \\
\hline \multirow[t]{3}{*}{ Crystal 2} & Peak max: & $94.7\left({ }^{\circ} \mathrm{C}\right)$ & Peak max: & $288.9\left({ }^{\circ} \mathrm{C}\right)$ & $\mathrm{T}:>350\left({ }^{\circ} \mathrm{C}\right)$ \\
\hline & Onset: & $88.4\left({ }^{\circ} \mathrm{C}\right)$ & Onset: & $285.2\left({ }^{\circ} \mathrm{C}\right)$ & $\Delta \mathrm{m}(\%): \quad-41.8$ \\
\hline & Offset: & $103.9\left({ }^{\circ} \mathrm{C}\right)$ & Offset: & $295.2\left({ }^{\circ} \mathrm{C}\right)$ & \\
\hline \multirow[t]{3}{*}{ Crystal 3} & Peak max: & $120.8\left({ }^{\circ} \mathrm{C}\right)$ & Peak max: & $294.8\left({ }^{\circ} \mathrm{C}\right)$ & $\mathrm{T}:>350\left({ }^{\circ} \mathrm{C}\right)$ \\
\hline & Onset: & $62\left({ }^{\circ} \mathrm{C}\right)$ & Onset: & $292.8\left({ }^{\circ} \mathrm{C}\right)$ & $\Delta \mathrm{m}(\%): \quad-42.8$ \\
\hline & Offset: & $142\left({ }^{\circ} \mathrm{C}\right)$ & Offset: & $298.5\left({ }^{\circ} \mathrm{C}\right)$ & \\
\hline \multirow[t]{3}{*}{ Crystal 4} & & & Peak max: & $316.3\left({ }^{\circ} \mathrm{C}\right)$ & $\mathrm{T}:>350\left({ }^{\circ} \mathrm{C}\right)$ \\
\hline & & & Onset: & $313.8\left({ }^{\circ} \mathrm{C}\right)$ & $\Delta \mathrm{m}(\%):-21.2$ \\
\hline & & & Offset: & $324.1\left({ }^{\circ} \mathrm{C}\right)$ & $\Delta \mathrm{m}(\%): \quad-3.46$ \\
\hline \multirow[t]{3}{*}{ Crystal 5} & Peak max: & $109.4\left({ }^{\circ} \mathrm{C}\right)$ & Peak max: & $263.6\left({ }^{\circ} \mathrm{C}\right)$ & $\mathrm{T}:>350\left({ }^{\circ} \mathrm{C}\right)$ \\
\hline & Onset : & $83\left({ }^{\circ} \mathrm{C}\right)$ & Onset : & $209.3\left({ }^{\circ} \mathrm{C}\right)$ & $\Delta \mathrm{m}(\%):-11.6$ \\
\hline & Offset : & $138\left({ }^{\circ} \mathrm{C}\right)$ & Offset : & $296.2\left({ }^{\circ} \mathrm{C}\right)$ & $\Delta \mathrm{m}(\%):-10.1$ \\
\hline
\end{tabular}



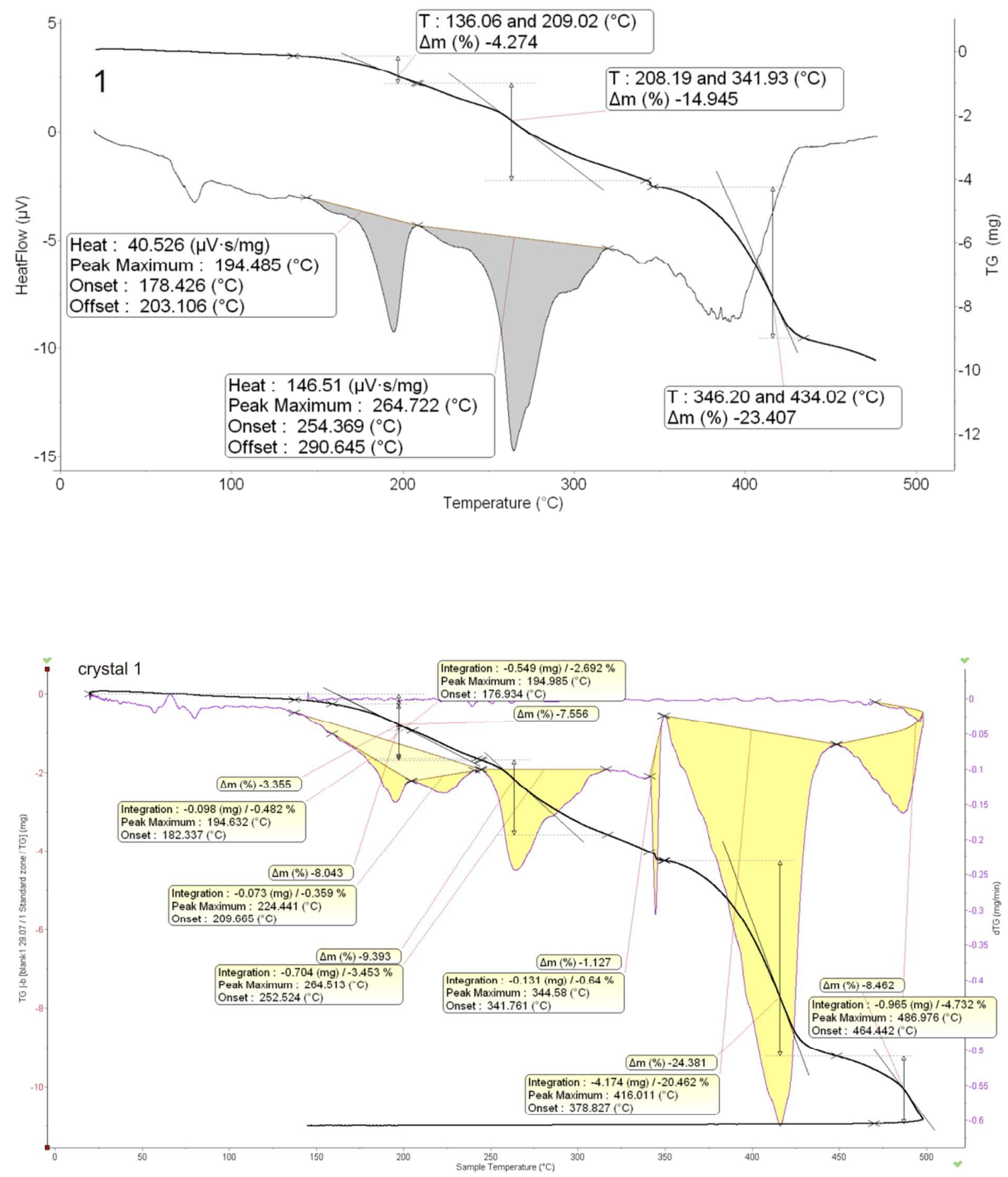

Figure S1 The TGA-DSC (a) and TG-DTG (b) plots for crystal 1 

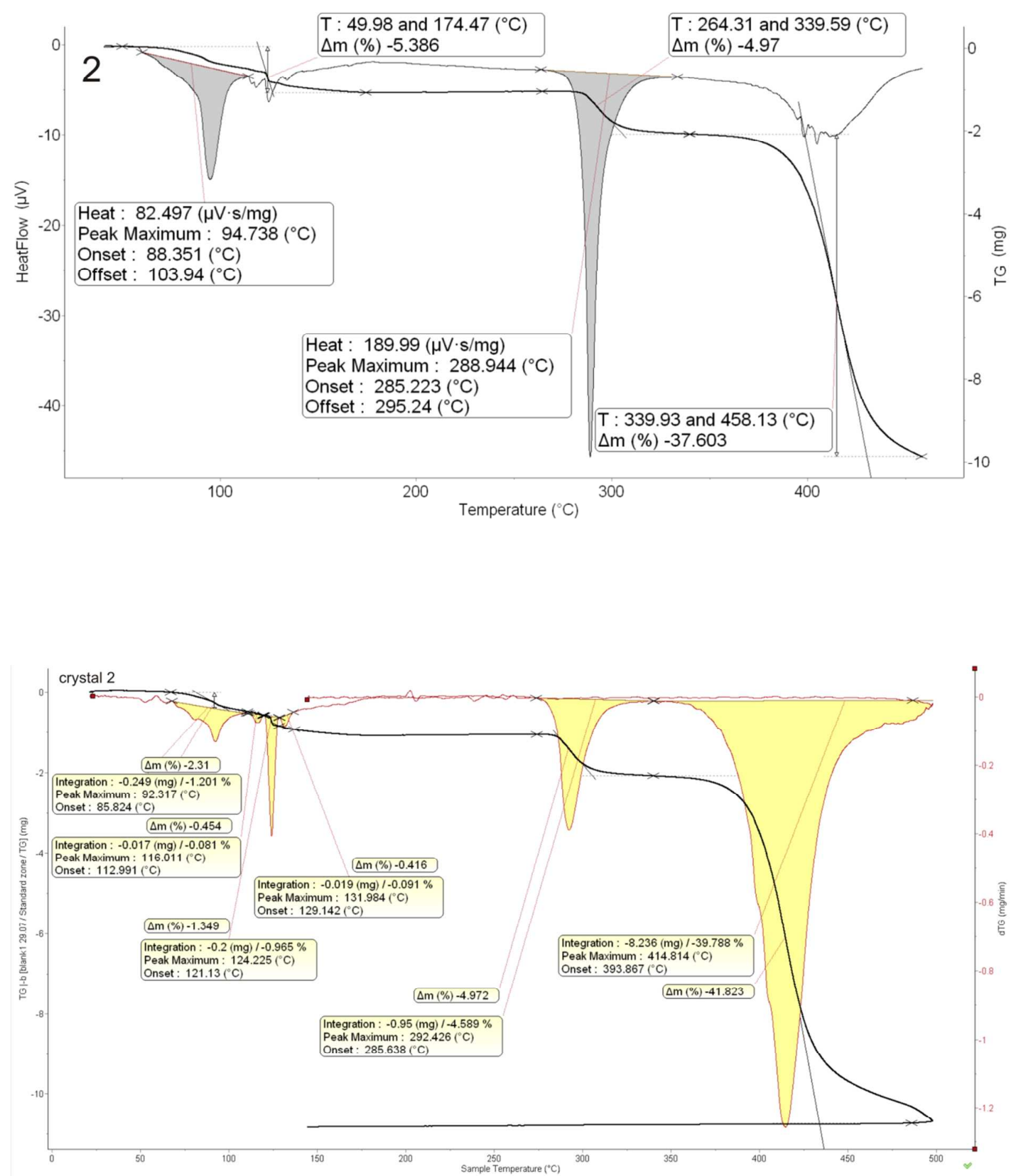

Figure S2 The TGA-DSC (a) and TG-DTG (b) plots for crystal 2. 

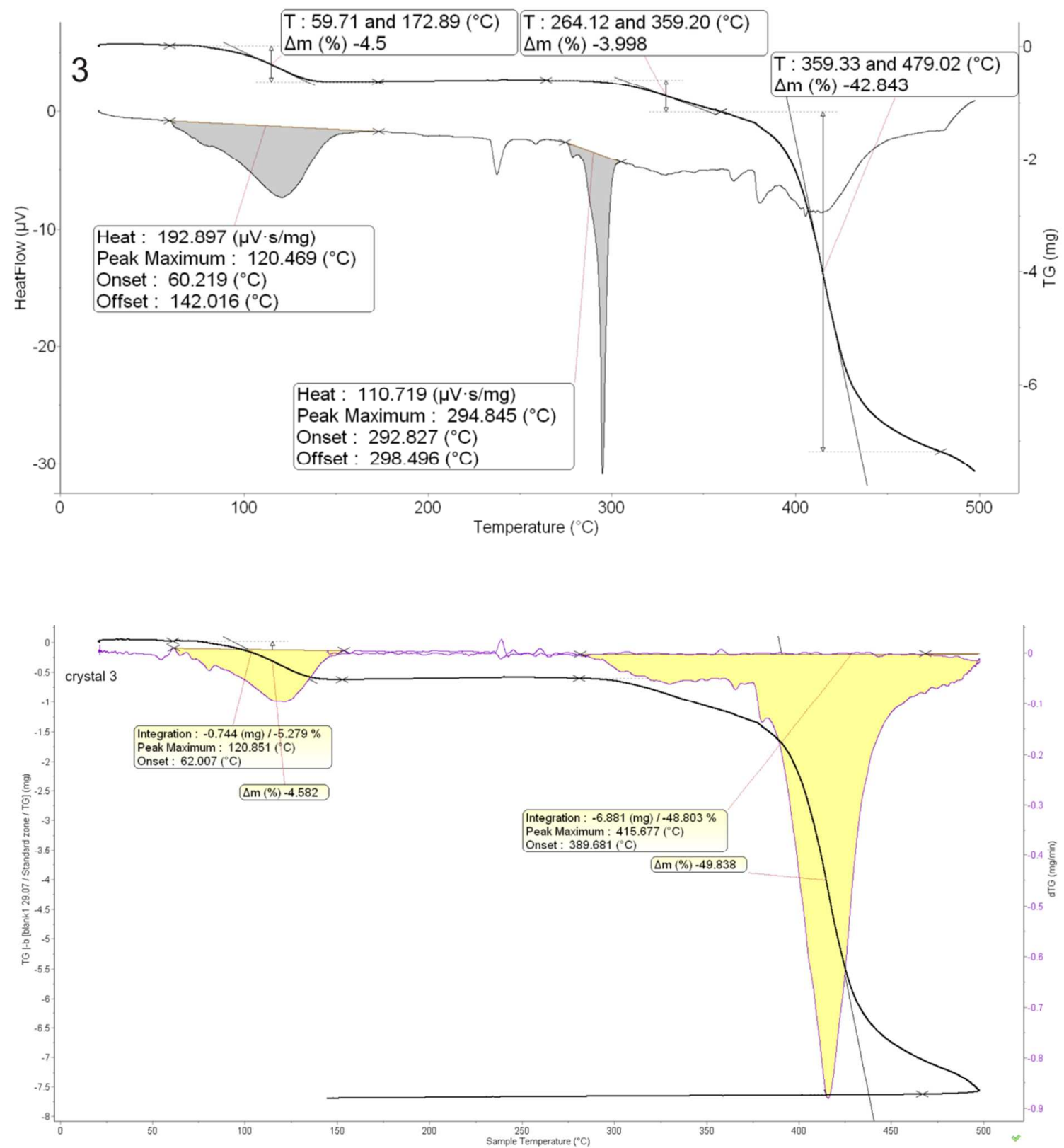

Figure S3. The TGA-DSC (a) and TG-DTG (b) plots for crystal 3. 

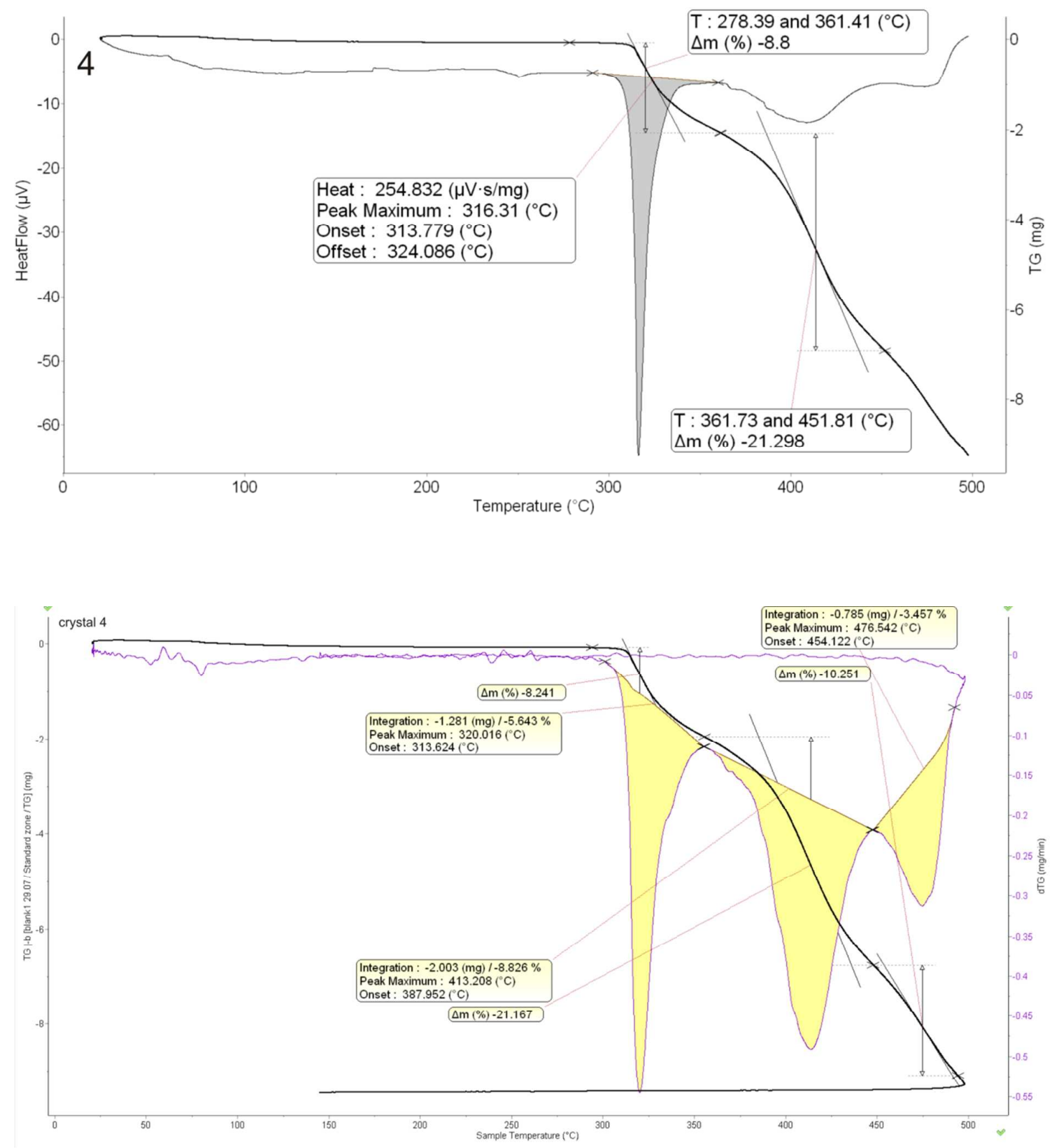

Figure S4. The TGA-DSC (a) and TG-DTG (b) plots for crystal 4. 

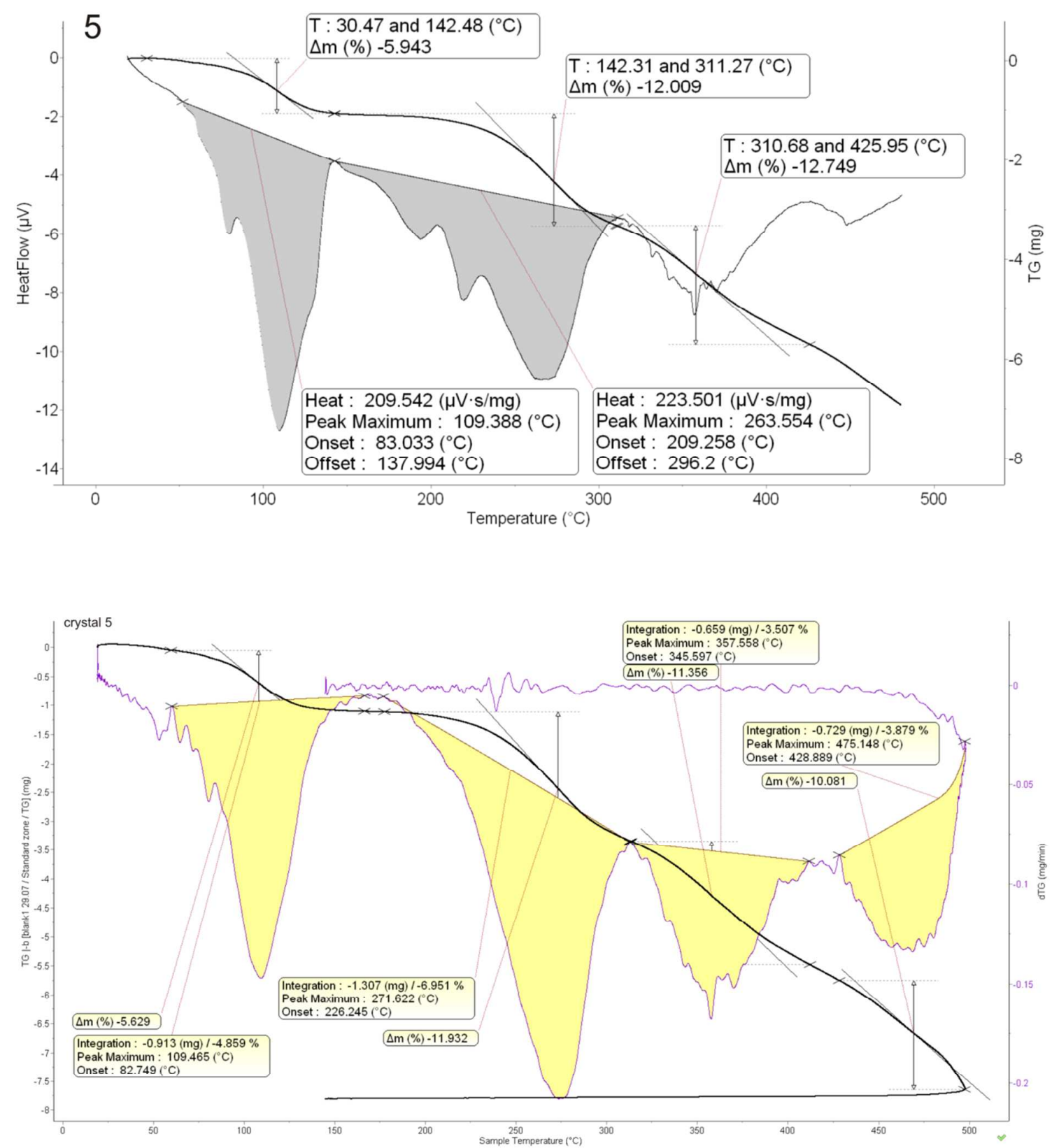

Figure S5. The TGA-DSC (a) and TG-DTG (b) plots for crystal 5. 


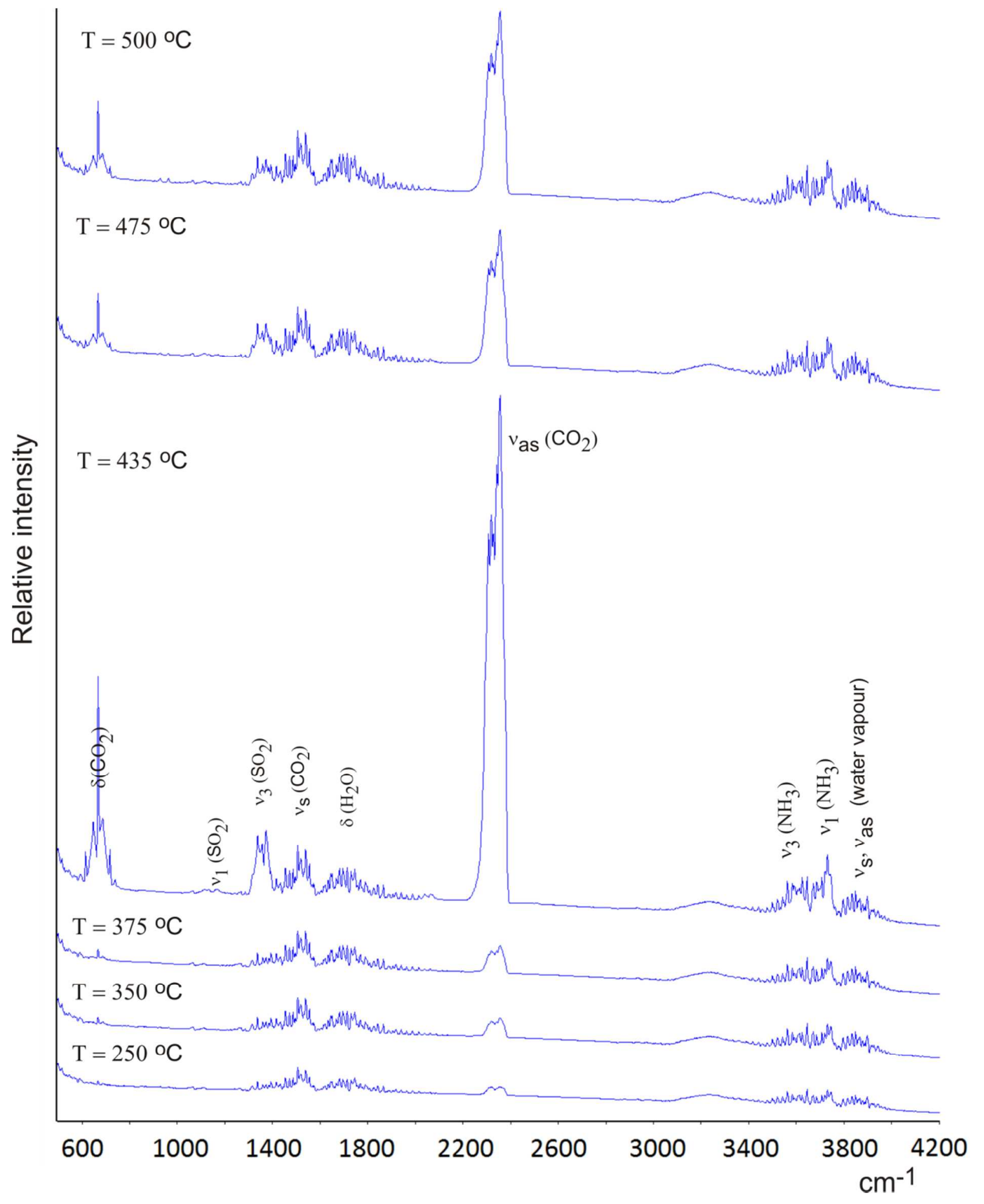

Figure S6. The TG-FTIR spectra of crystal 1 taken at temperatures of the endothermic changes 


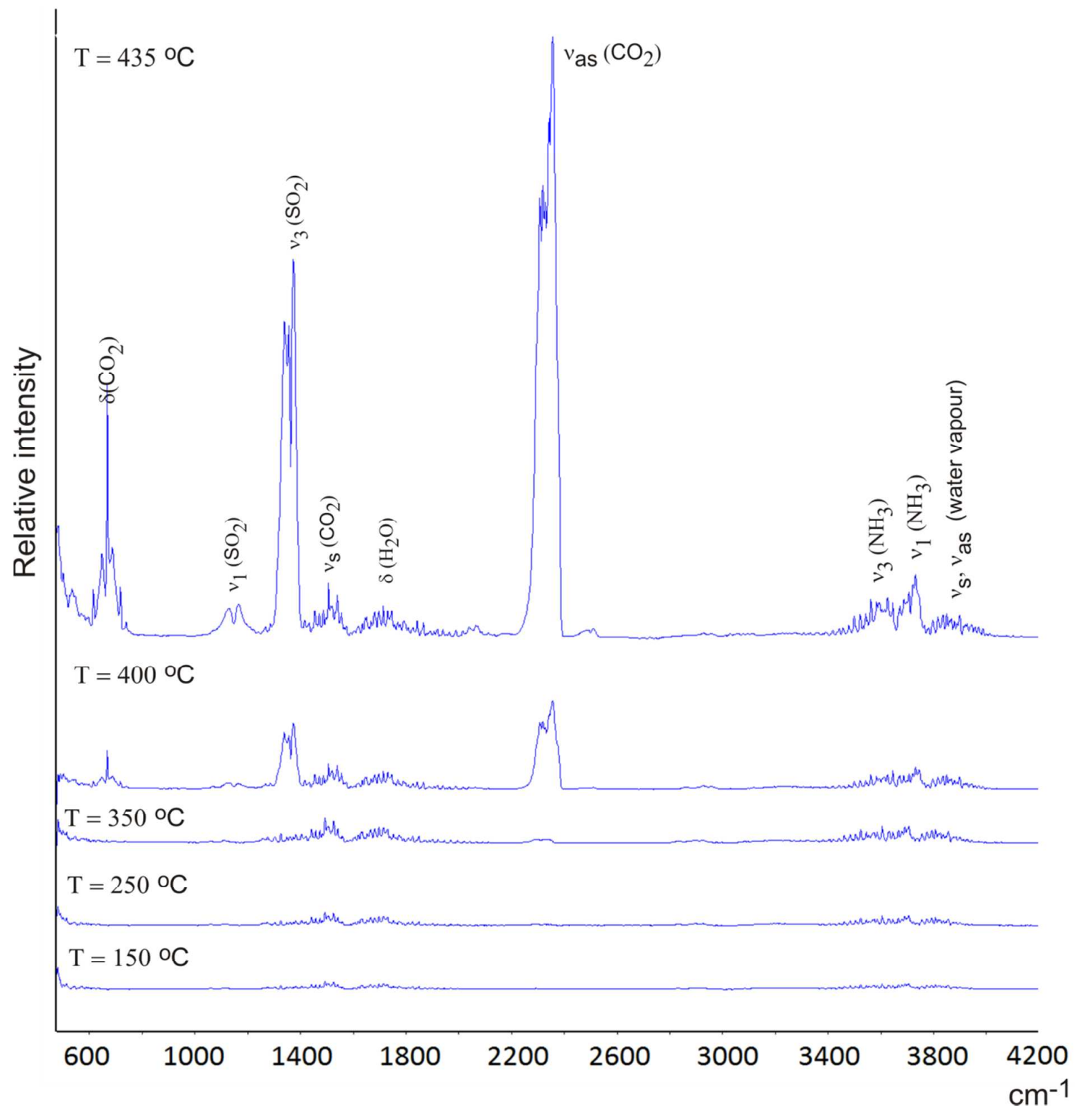

Figure S7. The TG-FTIR spectra of crystal 2 taken at temperatures of the endothermic changes 


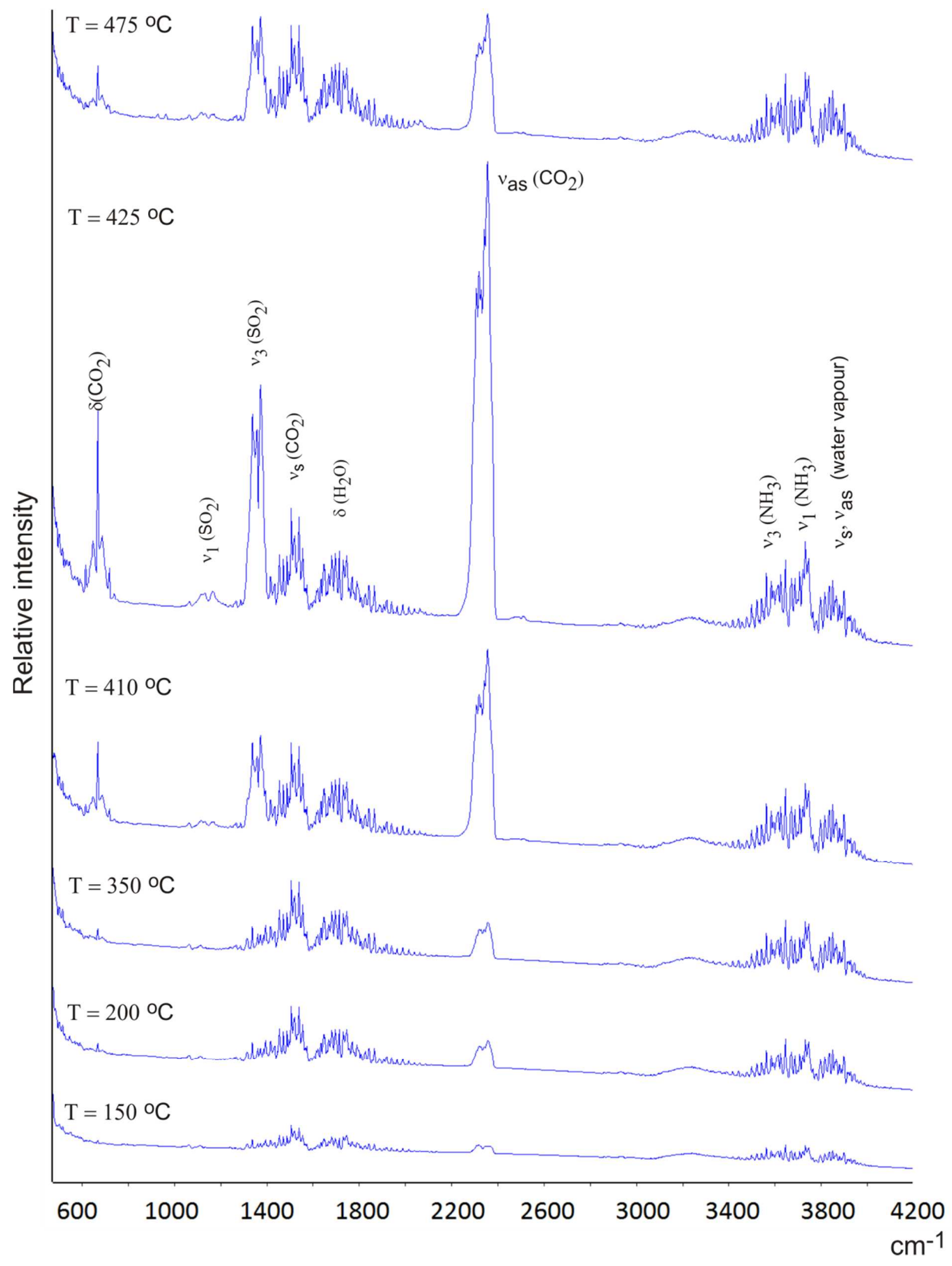

Figure S8. The TG-FTIR spectra of crystal 3 taken at temperatures of the endothermic changes 


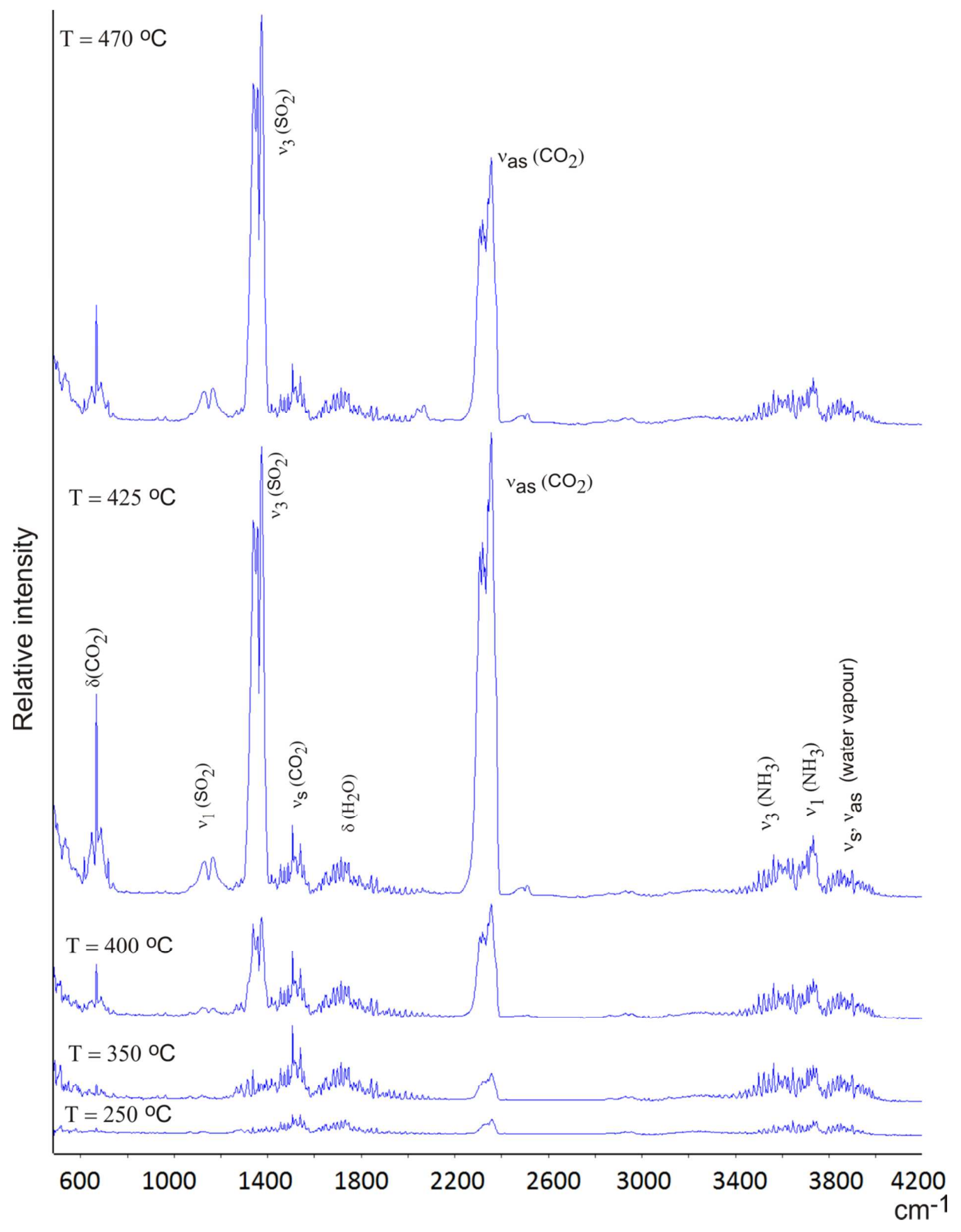

Figure S9. The TG-FTIR spectra of crystal 4 taken at temperatures of the endothermic changes. 


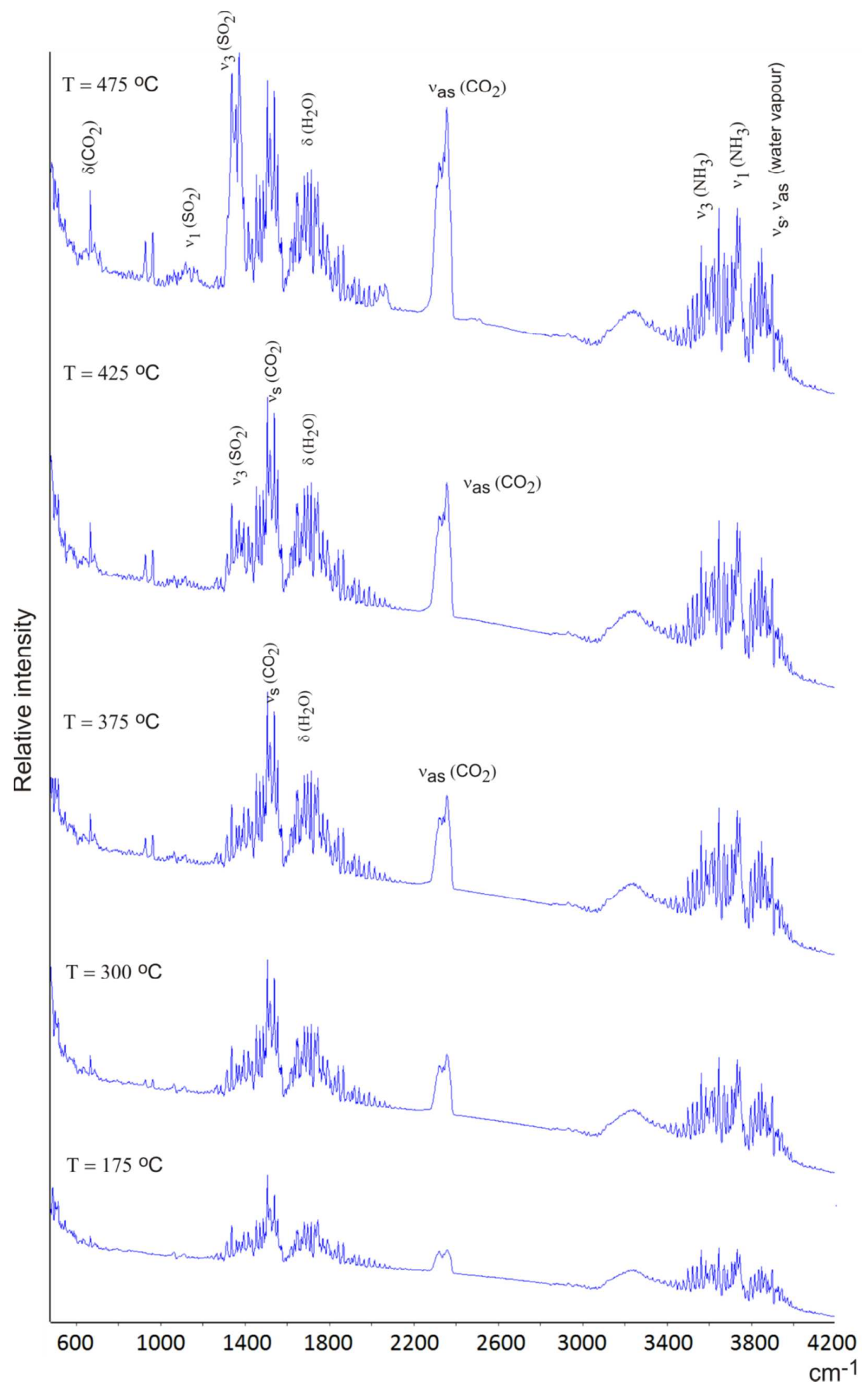

Figure S10. The TG-FTIR spectra of crystal 5 taken at temperatures of the endothermic changes. 
Table S2a. Internal vibrations of the functional groups

\begin{tabular}{|c|c|c|c|c|c|c|c|c|c|c|}
\hline \multirow[t]{2}{*}{$\begin{array}{c}\text { Type of } \\
\text { vibration }\end{array}$} & \multicolumn{2}{|c|}{$\begin{array}{c}\text { crystal } 1 \\
{\left[\mathrm{~cm}^{-1}\right]}\end{array}$} & \multicolumn{2}{|c|}{$\begin{array}{c}\text { crystal } 2 \\
{\left[\mathrm{~cm}^{-1}\right]}\end{array}$} & \multicolumn{2}{|c|}{$\begin{array}{c}\text { crystal } 3 \\
{\left[\mathrm{~cm}^{-1}\right]}\end{array}$} & \multicolumn{2}{|c|}{$\begin{array}{c}\text { crystal } 4 \\
{\left[\mathrm{~cm}^{-1}\right]}\end{array}$} & \multicolumn{2}{|c|}{$\begin{array}{c}\text { crystal } 5 \\
{\left[\mathrm{~cm}^{-1}\right]}\end{array}$} \\
\hline & IR & Ram. & IR & Ram. & IR & Ram. & IR & Ram. & IR & Ram. \\
\hline $\begin{array}{l}v(\mathrm{O}-\mathrm{H}) \\
\text { water }\end{array}$ & 3537 & & $\begin{array}{l}3448 \\
3354\end{array}$ & & $\begin{array}{l}3388 \\
3326\end{array}$ & & $\begin{array}{l}3440 \\
3341\end{array}$ & & $\begin{array}{l}3438 \\
3342\end{array}$ & \\
\hline$v \mathrm{NH}_{2}$ & $\begin{array}{l}3305 \\
3142\end{array}$ & & $\begin{array}{l}3235 \\
3142 \\
3112 \\
\end{array}$ & & $\begin{array}{l}3194 \\
3142\end{array}$ & & $\begin{array}{l}3219 \\
3110\end{array}$ & & $\begin{array}{l}3191 \\
3109\end{array}$ & \\
\hline$v(\mathrm{Npy}-\mathrm{H})$ & $\begin{array}{l}2898 \\
2843\end{array}$ & & $\begin{array}{l}2905 \\
2868 \\
2840\end{array}$ & & $\begin{array}{l}2967 \\
2852 \\
2821\end{array}$ & & 2924 & & 2804 & \\
\hline$v(C) O-H$ & 2572 & & 2677 & & $\begin{array}{l}2681 \\
2551\end{array}$ & & 2701 & & & \\
\hline$v(C=O)$ & $\begin{array}{l}1725 \\
1666\end{array}$ & 1726 & $\begin{array}{l}1728 \\
1700\end{array}$ & 1724 & $\begin{array}{l}1720 \\
1700\end{array}$ & $\begin{array}{l}1730 \\
1709\end{array}$ & $\begin{array}{l}1729 \\
1698\end{array}$ & 1730 & $\begin{array}{l}1728 \\
1698\end{array}$ & 1712 \\
\hline $\begin{array}{l}\delta\left(\mathrm{NH}_{2}\right) \\
\delta(\mathrm{O}-\mathrm{H})\end{array}$ & $\begin{array}{l}1408 \\
1382 \\
1326\end{array}$ & $\begin{array}{l}1386 \\
1330\end{array}$ & $\begin{array}{l}1401 \\
1355 \\
1335\end{array}$ & 1403 & 1399 & 1384 & $\begin{array}{l}1402 \\
1377 \\
1320\end{array}$ & $\begin{array}{l}1398 \\
1319\end{array}$ & $\begin{array}{l}1403 \\
1378 \\
1321\end{array}$ & $\begin{array}{l}1395 \\
1376\end{array}$ \\
\hline $\begin{array}{l}v(C-O) \\
v(C-O)\end{array}$ & 1294 & & 1289 & 1287 & $\begin{array}{l}1299 \\
1265 \\
\end{array}$ & 1288 & 1286 & 1280 & 1301 & 1302 \\
\hline$v(S=0)$ & $\begin{array}{l}1235 \\
1173 \\
1111\end{array}$ & $\begin{array}{l}1252 \\
1161 \\
1132\end{array}$ & $\begin{array}{l}1225 \\
1185 \\
1155 \\
1111 \\
\end{array}$ & $\begin{array}{l}1177 \\
1140\end{array}$ & $\begin{array}{l}1245 \\
1186 \\
1153 \\
1111 \\
\end{array}$ & $\begin{array}{l}1222 \\
1188 \\
1138\end{array}$ & $\begin{array}{l}1220 \\
1184 \\
1158 \\
1116 \\
\end{array}$ & $\begin{array}{l}1220 \\
1181 \\
1143 \\
1125 \\
\end{array}$ & $\begin{array}{l}1220 \\
1184 \\
1160 \\
1116 \\
\end{array}$ & $\begin{array}{l}1176 \\
1122\end{array}$ \\
\hline$v(C-O)$ & 1043 & 1048 & 1046 & 1049 & 1044 & 1047 & 1044 & 1044 & 1044 & 1044 \\
\hline$v(C-S)$ & 997 & 998 & 996 & 998 & 997 & 998 & 997 & 998 & 996 & 997 \\
\hline $\begin{array}{l}\omega\left(\left(\mathrm{NH}_{2}\right)\right. \\
\text { def. vib. of } \\
\mathrm{SO}_{3}\end{array}$ & $\begin{array}{l}669 \\
625 \\
575 \\
545 \\
512\end{array}$ & $\begin{array}{l}626 \\
556\end{array}$ & $\begin{array}{l}673 \\
623 \\
572 \\
528 \\
488 \\
\end{array}$ & $\begin{array}{l}627 \\
547\end{array}$ & $\begin{array}{l}674 \\
613 \\
542 \\
499\end{array}$ & $\begin{array}{l}647 \\
528\end{array}$ & $\begin{array}{l}674 \\
623 \\
568 \\
547\end{array}$ & $\begin{array}{l}570 \\
539\end{array}$ & $\begin{array}{l}674 \\
622 \\
567\end{array}$ & $\begin{array}{l}566 \\
542\end{array}$ \\
\hline
\end{tabular}


Table S2b. Internal vibrations of the aromatic rings

\begin{tabular}{|c|c|c|c|c|c|c|c|c|c|c|}
\hline \multirow[t]{2}{*}{$\begin{array}{l}\text { Type of } \\
\text { vibration }\end{array}$} & \multicolumn{2}{|c|}{$\begin{array}{c}\text { crystal } 1 \\
{\left[\mathrm{~cm}^{-1}\right]}\end{array}$} & \multicolumn{2}{|c|}{$\begin{array}{c}\text { crystal } 2 \\
{\left[\mathrm{~cm}^{-1}\right]}\end{array}$} & \multicolumn{2}{|c|}{$\begin{array}{c}\text { crystal } 3 \\
{\left[\mathrm{~cm}^{-1}\right]}\end{array}$} & \multicolumn{2}{|c|}{$\begin{array}{c}\text { crystal } 4 \\
{\left[\mathrm{~cm}^{-1}\right]}\end{array}$} & \multicolumn{2}{|c|}{$\begin{array}{c}\text { crystal } 5 \\
{\left[\mathrm{~cm}^{-1}\right]}\end{array}$} \\
\hline & IR & Ram. & IR & Ram. & IR & Ram. & IR & Ram. & IR & Ram. \\
\hline$v(\mathrm{C}-\mathrm{H})$ & $\begin{array}{l}3098 \\
2952\end{array}$ & 3087 & $\begin{array}{l}3079 \\
3037 \\
2981\end{array}$ & 3082 & $\begin{array}{l}3095 \\
3078 \\
3041\end{array}$ & 3102 & $\begin{array}{l}3074 \\
3003\end{array}$ & $\begin{array}{l}3095 \\
3075\end{array}$ & $\begin{array}{l}2999 \\
2918\end{array}$ & 3095 \\
\hline$v(C-C)$ & $\begin{array}{l}1622 \\
1546\end{array}$ & $\begin{array}{l}1609 \\
1590 \\
1550\end{array}$ & $\begin{array}{l}1644 \\
1609 \\
1570\end{array}$ & $\begin{array}{l}1628 \\
1603\end{array}$ & $\begin{array}{l}1652 \\
1597 \\
1530\end{array}$ & $\begin{array}{l}1643 \\
1604 \\
1536\end{array}$ & $\begin{array}{l}1660 \\
1606\end{array}$ & $\begin{array}{l}1644 \\
1607 \\
1564\end{array}$ & $\begin{array}{l}1657 \\
1554\end{array}$ & $\begin{array}{l}1644 \\
1603 \\
1562\end{array}$ \\
\hline$v(C=N)$ & $\begin{array}{l}1476 \\
1454\end{array}$ & 1488 & $\begin{array}{l}1492 \\
1440\end{array}$ & 1490 & 1457 & 1423 & $\begin{array}{l}1491 \\
1446\end{array}$ & $\begin{array}{l}1498 \\
1457\end{array}$ & 1491 & 1491 \\
\hline $\begin{array}{l}\text { Def. } \\
\text { vibrations }\end{array}$ & $\begin{array}{l}915 \\
878 \\
847\end{array}$ & 850 & $\begin{array}{l}911 \\
895 \\
869\end{array}$ & $\begin{array}{l}899 \\
841\end{array}$ & $\begin{array}{l}921 \\
899 \\
806\end{array}$ & $\begin{array}{l}901 \\
852\end{array}$ & $\begin{array}{l}917 \\
851 \\
826\end{array}$ & 900 & $\begin{array}{l}915 \\
851 \\
832\end{array}$ & \\
\hline $\begin{array}{l}\text { Twisting } \\
\text { vibrations }\end{array}$ & $\begin{array}{l}760 \\
721\end{array}$ & 721 & $\begin{array}{l}793 \\
757 \\
717\end{array}$ & 716 & $\begin{array}{l}756 \\
715\end{array}$ & $\begin{array}{l}783 \\
715\end{array}$ & $\begin{array}{l}774 \\
755 \\
714\end{array}$ & $\begin{array}{l}756 \\
712\end{array}$ & $\begin{array}{l}774 \\
755 \\
705\end{array}$ & $\begin{array}{l}759 \\
712\end{array}$ \\
\hline
\end{tabular}

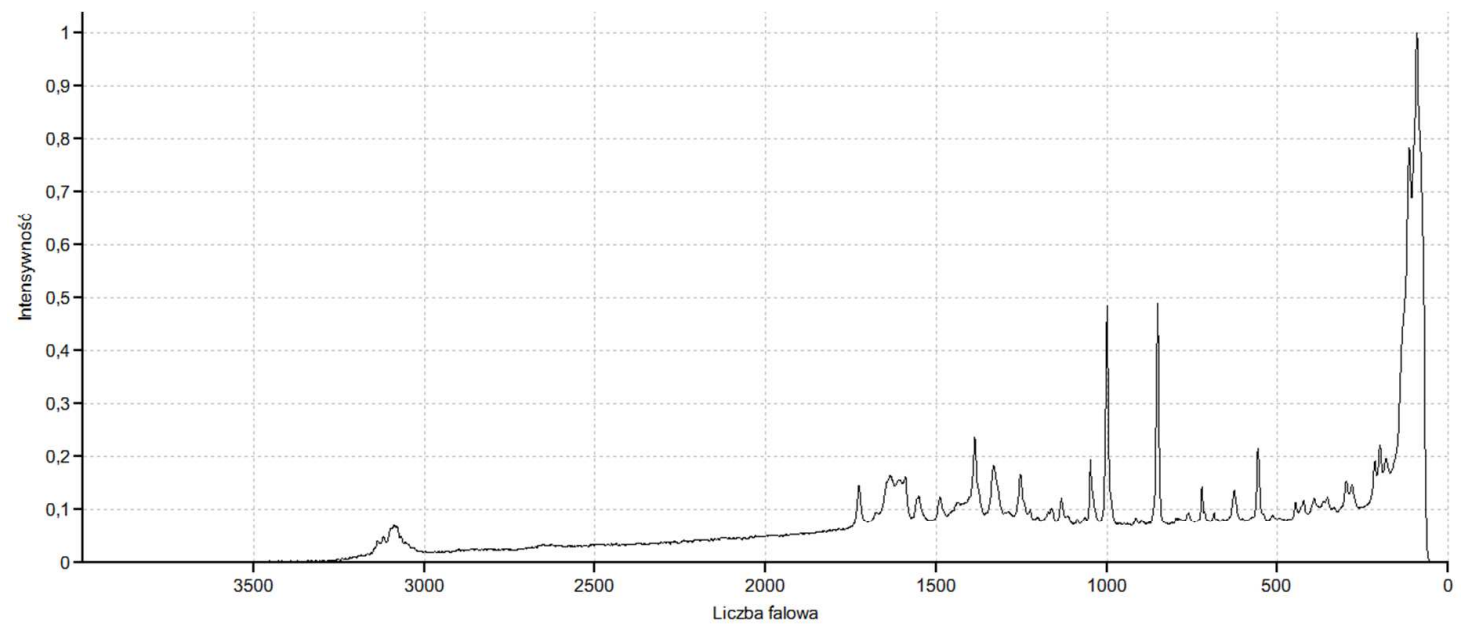

Figure S11. The Raman spectrum of crystal 1 


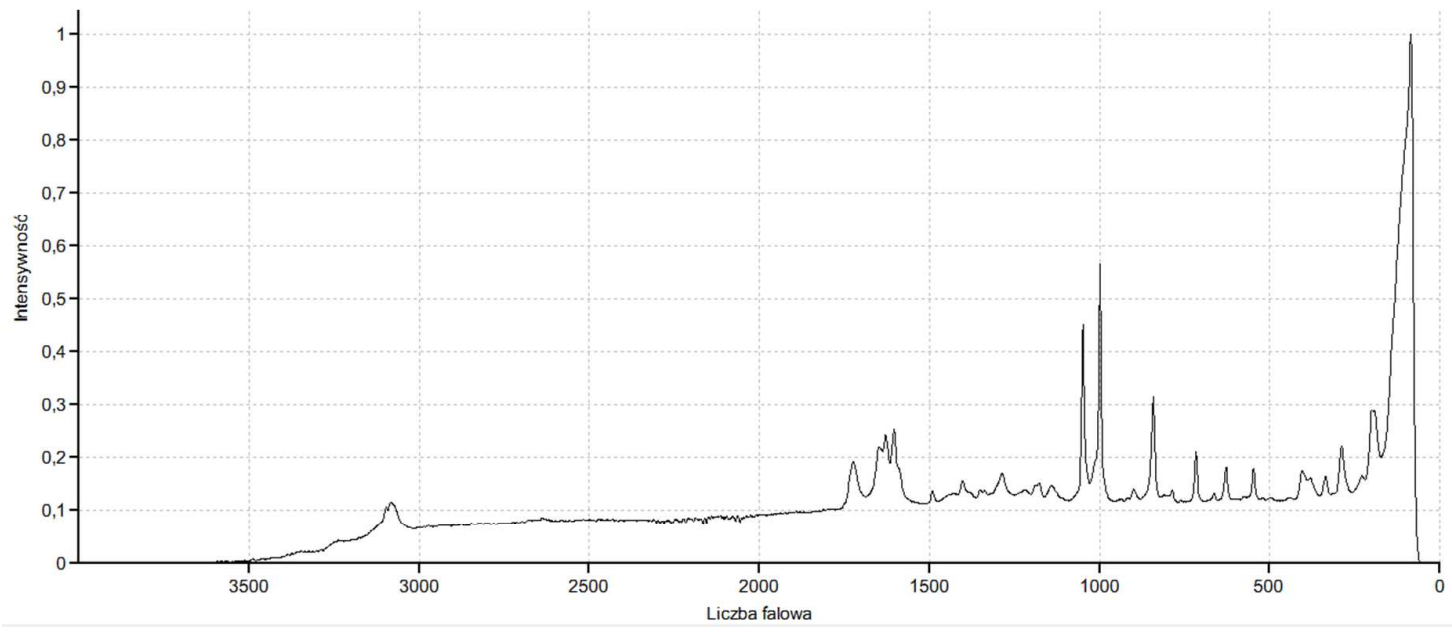

Figure S12. The Raman spectrum of crystal 2

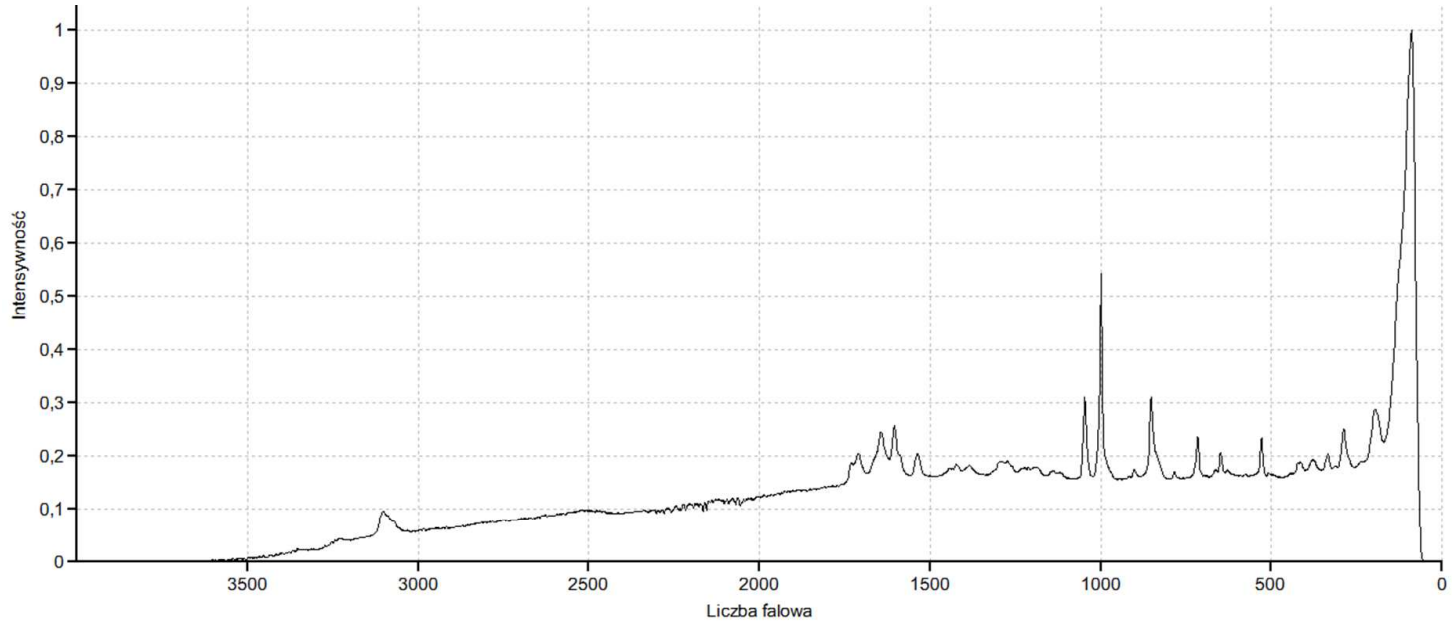

Figure S13. The Raman spectrum of crystal 3 


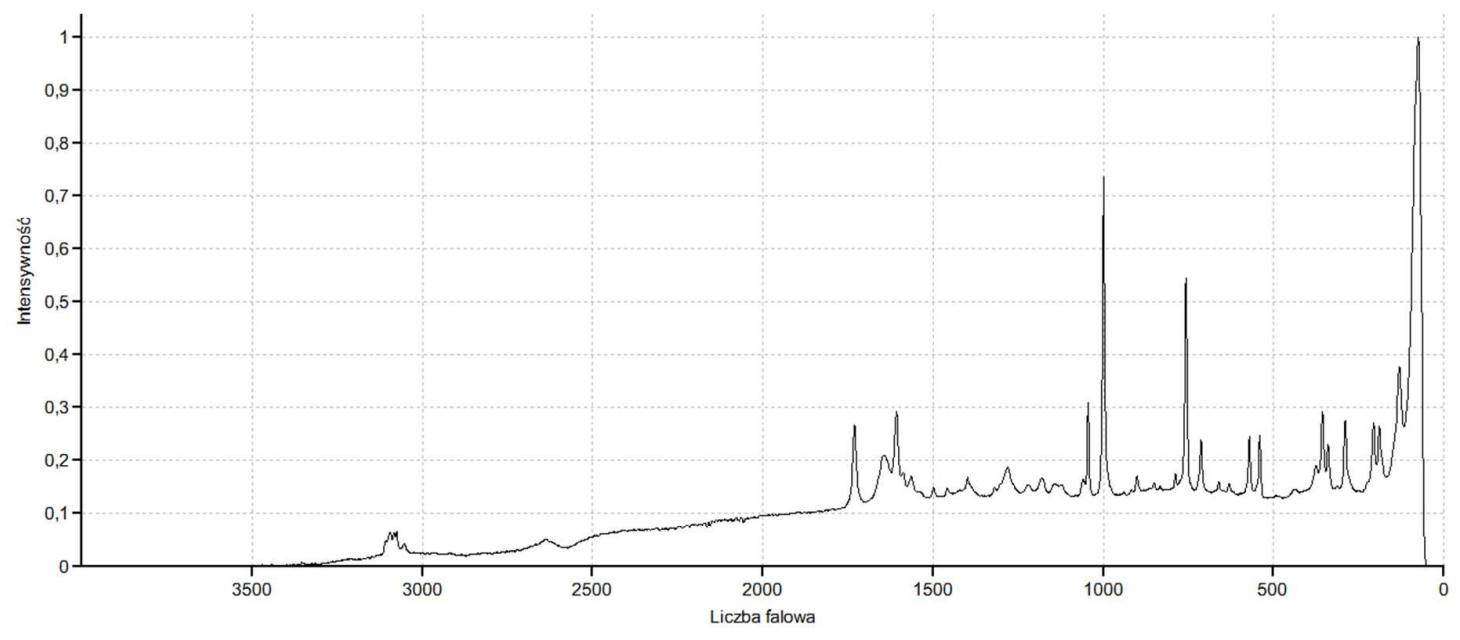

Figure S114. The Raman spectrum of crystal 4

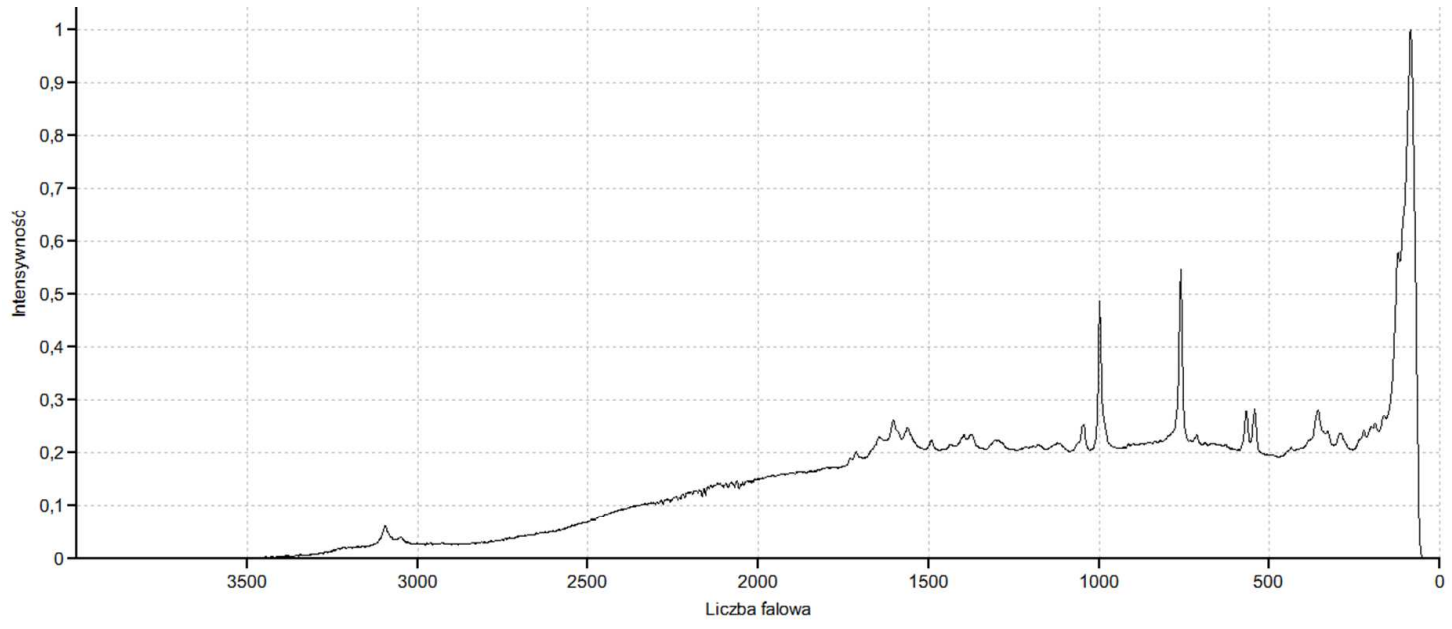

Figure S15. The Raman spectrum of crystal 5 